\title{
Till Thimme, Hubertus Deimel, Gerd Hölter: Bewegung und psychische Gesundheit von Kindern und Jugendlichen. Grundlagen - Störungsbilder - Therapie
}

Schattauer, 2021, Stuttgart, 496 Seiten, 58,00€ (D)

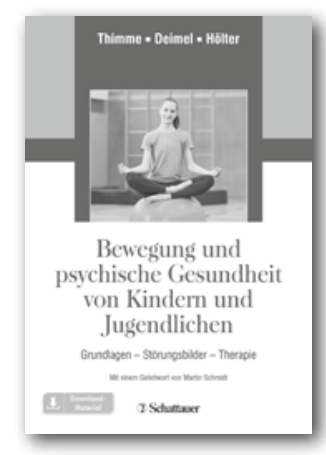
ten zuordnen als dem therapeutischen Bereich. Aber schon in der Ankündigung teilen die Autoren und der Verlag mit, dass es sich um das erste umfassende Buch zur Bewegungstherapie mit Kindern und Jugendlichen im deutschsprachigen Raum handelt. Und das macht neugierig!

Doch bereits durch diese sicherlich beeindruckende Werbeaufmachung stellt sich für die Autoren die erste große Hürde: Welche Form der Bewegungstherapie meinen sie? Motopädie, Motologie, Psychomotorik, Gymnastik, Physiotherapie, Sport, um nur einige Begrifflichkeiten zu nennen? Kaum ein Begriff wird im deutschsprachigen Raum im Rahmen gesundheits- und krankheitsintervenierender Maßnahmen so vieldeutig verwendet wie Bewegungstherapie. Das beansprucht der Masseur in seiner Ausbildung als rein funktionalmotorische Methode wie der Physiotherapeut, ebenso der Sportwissenschaftler bis hin zum Körperpsychotherapeuten, der den Bewegungsbegriff wiederum ganz anders interpretiert. Folgerichtig widmen sich die Autoren in ihrem ersten Kapitel zunächst den Grundlagen und theoretischen Orientierungen mit dem Verständnis der Bewegung als elementares Phänomen von Entwicklung, Erleben und Verhalten mit dem Ziel, Bewegungstherapie als eigenständiges Verfahren zu definieren.

Mir gefällt sehr der zunächst dargestellte geschichtliche Abriss über die Bewegungstherapie bei psychisch erkrankten Kindern und Jugendlichen, der beginnend von der rein funktionalen Behandlung über die Psychomotorik nach Kiphard führt und schließlich mit

der dritten Facette der Bewegungstherapie, orientiert an psychotherapeutischen Theorien, die Breite des Denkens aufzeigt. Bereits hier zeigt sich das Verständnis der Autoren von ihrem bewegungstherapeutischen Begriff in der Therapie mit Kindern, der sich eher an ein funktional-motorisches Verständnis mit erlebnisorientierten Inhalten und weniger an ein psychotherapeutisches Bewegungsparadigma hält. Damit entwickeln sie zunächst die Bewegungstherapie als ein eigenständiges Verfahren, das mit geeigneten Mitteln von Bewegung, Spiel und Sport auf die Linderung von Störungen mit Krankheitswert und auf die Wiederherstellung und Verbesserung der Gesundheit abzielt (S.71). Sie sehen die Bewegungstherapie im Schnittpunkt von Bewegungs- und Sportwissenschaft, Medizin und Psychologie. Dementsprechend werden in diesem ersten Kapitel Bezüge zu Rahmentheorien wie der Neurobiologie, Salutogenesekonzepten, Bezugssystemen wie dem ICF und zur Evidenzbasierung hergestellt. Gerade junge TherapeutInnen erhalten hier in kurzer Form einen wichtigen Überblick über einige Themen, die heute im aktuellen Kontext von verschiedenen bewegungstherapeutischen Richtungen diskutiert werden. Konsequent verfolgen die Autoren über das gesamte Buch ihr Anliegen, die Bewegungstherapie evidenzbasiert darzustellen bzw. kritisch zu beleuchten. Der Mangel an Wirksamkeitsstudien bei einigen Störungsbildern wird auch hier deutlich, dennoch ist anzumerken, dass gute Methoden und auch Verfahren nicht dadurch besser oder schlechter geworden sind, weil eine RCT-Studie deren Wirksam- 
keit bestätigt hat. Der kritische Diskurs in der „evidence based practice“ ist hier gefragt.

Im folgenden zweiten Kapitel werden Ziele, Inhalte und Settings dargestellt. Hier erhält der/ die Leserin eine differenzierte Auflistung von Therapiezielen, erfährt vielfältige inhaltliche Möglichkeiten über das Trainieren, Formen der Erlebnispädagogik, Entspannung, Achtsamkeit, Einbeziehung von Kampfkünsten bis zu einem Exkurs zur pferdegestützten Therapie. Was noch in dieser Vielfalt fehlt, wäre Natur- oder Waldtherapie. In den Settings werden u.a. die Klinik, ambulante Hilfe, Schulsystem und weitere dargestellt.

Kapitel 3 war für mich das Spannendste, denn hier geht es um die wichtigsten psychischen Erkrankungen im Kindes- und Jugendalter. Damit nimmt dieses Kapitel fast die Hälfte des gesamten Buches ein und beschreibt in störungsspezifischen Teilkapiteln die Aufmerksamkeitsdefizit- und Hyperaktivitätsstörungen, Störungen des Sozialverhaltens, Angststörungen, depressive Störungen, Essstörungen, Störungen durch Substanzmissbrauch, Schizophrenie und schließlich Autismus-Spektrum-Störungen. Dies wird sehr systematisch über eine Einstimmung zum Störungsbild vorgenommen, wichtigen klinischen Informationen wie Definitionen, Klassifikation, Symptome, zur Epidemiologie, Ätiologie und schließlich zur Therapie. Die Ziele der Bewegungstherapie des Autorenteams werden jeweils im motorisch-sensorischen Bereich, im kognitiven Bereich, im emotionalen Bereich und im sozialen Bereich verankert. Methodische Hinweise wie die Motivationslage der PatientInnen, Rahmenbedingungen, Beziehung und Umgang ergänzen diese Darstellung.

Abschließend wird die aktuelle Forschungslage erörtert. Eine Zusammenfassung gibt über alle Punkte eine kurze Übersicht. Die Systematik des Vorgehens in diesem Kapitel macht das Buch besonders wertvoll zum einen für TherapeutInnen, die in diesen Arbeitsbereich einsteigen, natürlich für Auszubildende, aber auch für leitende Ärztlnnen und für verwandte Berufsgruppen. Hier erfährt der / die Leserln, wie das Autorenteam konkret arbeitet, was die Bewegungstherapie leisten kann (z.B. im Team) und - eben auch - was nicht. Ich finde, dieses Kapitel hat höchsten Informationswert und gibt sehr übersichtlich den aktuellen Wissenstand zu den Störungsbildern wieder. Schon allein dadurch hat dieses Buch einen festen Platz im Bücherregal, gerade wenn man mit Kindern und Jugendlichen arbeitet.

Das eigentlich schon letzte Kapitel 4 bietet dann den Praktikerlnnen wertvolle Hilfen für den bewegungstherapeutischen Alltag, z. B. wie man Therapiestunden vorbereitet, Hilfen bei der Durchführung und Hilfen zur Nachbereitung. Die Hilfen für die TherapeutInnen, z.B. die Erwähnung von Supervision, sind etwas kurz geraten. Auch vermisse ich spezifische Ausführungen zur therapeutischen Beziehung und Grundhaltung. Diese wird zwar in einigen Unterkapiteln immer wieder erwähnt oder angedeutet, spielt aber möglicherweise aus Sicht der Autoren nicht diese herausragende Rolle wie in körperpsychotherapeutischen Methoden. Das lässt sich aber auch aus dem Anliegen der Autoren herauslesen, weil sie bewusst ein Buch für die aktuelle Bewegungstherapie in der Tradition von Psychomotorik, Sportwissenschaft mit erweiterten theoretischen Bezugspunkten in Richtung auf psychologische und psychotherapeutische Theorien geschrieben haben (S.20) und für sie dadurch die Grenzen zu einer Form der Psychotherapie unschärfer werden. Das ist der Spagat des Buches: seelische Erkrankungen bei Kindern und Jugendlichen mit bewegungstherapeutischen Techniken und Methoden zu behandeln, ohne typisch psychotherapeutische, sprich körper- oder leibpsychotherapeutische Interventionen vor dem Hintergrund eines psychotherapeutischen Paradigmas einzusetzen. Die Abgrenzung der Autoren wird hierbei auch ganz klar formuliert: Sie formulieren dass in der Weiterbildung auch von funk- 
tional und erlebniszentriert tätigen Therapeuten, für die dieses Buch geschrieben ist, Weiterbildungen bei körperorientierten, psychotherapeutischen Methoden - und damit ist sicherlich die Konzentrative Bewegungstherapie, die Integrative Bewegungstherapie, Tanztherapie, Körperpsychotherapie und weitere Methoden gemeint - auf ein großes Interesse stoßen (S. 21). Und ich stimme der Auffassung der Autoren vollumfänglich zu, dass eine Doppelqualifikation Bewegungs- / PsychotherapeutIn die klinische Versorgung sicherlich bereichern kann. Um die Kritik auch mal umzudrehen: Auch bei KörperpsychotherapeutInnen werden funktional übungszentrierte Techniken und Methoden wie Lauftherapie und Ausdau- ertherapie, z.B. bei Depression und Schmerz, nach wie vor zu wenig eingesetzt.

Das Autorenteam Thimme, Deimel und Hölter legt wirklich ein fundiertes, differenziertes und akribisch ausgearbeitetes Werk zur motorisch-funktionellen und erlebniszentrierten Bewegungstherapie für Kinder und Jugendliche auf dem neuesten Wissenschaftsstand vor. Es wird die Bewegungstherapie bekannter machen, und man kann es allen Fachgruppen, die mit Kindern und Jugendlichen arbeiten, sehr empfehlen.

Martin J. Waibel

DOI 10.2378/ktb2021.art21d

\section{Jonas A. Hamm: Trans* und Sex. Gelingende Sexualität zwischen Selbstannahme, Normüberwindung und Kongruenzerleben}

Psychosozial-Verlag, 2020, Gießen, 147 Seiten, 19,90€ (D)

\section{L} ängst überfällig, dieses Buch! Was Jonas A. - Hamm unter gelingender Sexualität in seinem Buch „Trans* und Sex“ aufschlüsselt, ist für viele Menschen noch nicht selbstverständlich und somit ein enorm wichtiger Beitrag zur Ent-Tabuisierung von (Trans*-)Sexualität. Fein und ausdifferenziert beobachtend zeigt Hamm die große Lücke auf zwischen gesellschaftlicher Realität, wissenschaftlicher Aufarbeitung und persönlicher Erfahrung von gelebter Sexualität. Das realisiert er über eine qualitative Studie mit sechs sorgfältig ausgewählten und reflektierten Personen einer Minderheit, die die sehr konkrete, sexuelle Realität von Trans*Personen zeigt, welche keine genitalangleichende/n Operation/en anstreben.

Sowohl für mich persönlich, der auch zu dieser Minderheit gehört, als auch für viele meiner KlientInnen hat dieses Buch die Bedeutung eines Meilensteins in seiner Perspektive von / auf und für Trans*-Men-

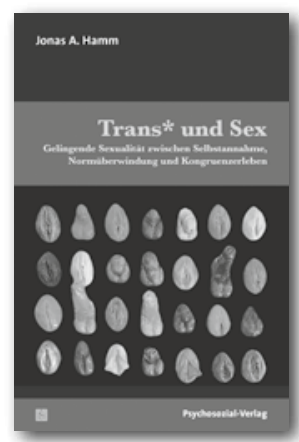
schen mit dem Ziel ihrer Selbstbestimmung über den eigenen Körper.

Als systemischer Berater, Somatiker und Sexological Bodyworker begleite ich Trans*Menschen darin, ihre Verbindung zum eigenen Körper zu stärken. Dabei ist meine Erfahrung, dass es besonders unterstützend ist, vor allem die Aspekte der gelingenden Sexualität in den Fokus gestellt zu wissen. Diese, wie in diesem Werk noch mal ausdifferenziert betrachtet, schaffen unter anderem Anregung zur Selbstreflexion, sind aber vor allem für die Begleitprozesse in Bezug auf eigene internalisierte Vorstellungen wertvoll. Die Grundfrage „Was brauchst du, um mit deiner eigenen Sexualität zufrieden zu sein, mit deinem eigenen Körper 